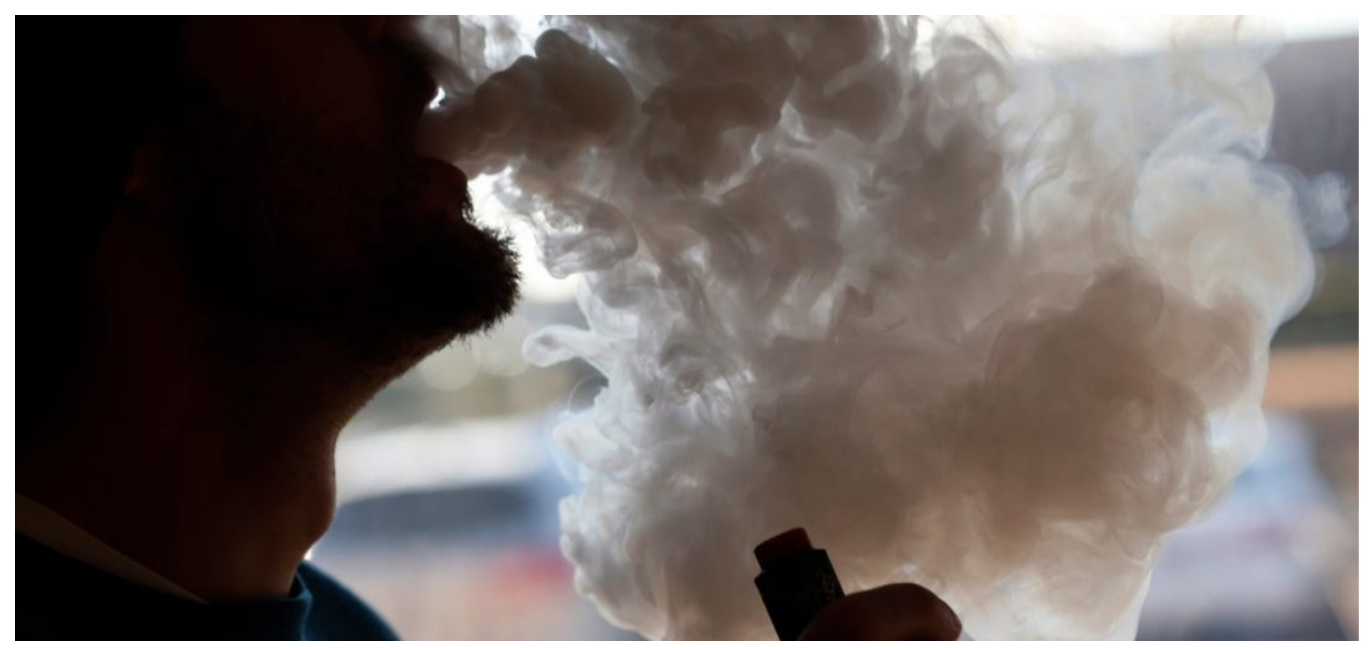

\title{
Use of Nicotine Replacement Therapy and Behavioral Support to Quit ENDS
}

\author{
Michelle Sahr ${ }^{1}$, Noah Blower ${ }^{1}$, Shelby Kelsh
}

1 Ferris State University

Funding: The author(s) received funding from the Michigan Pharmacists Foundation and Ferris State University Faculty Research Grant to support this work.

Potential competing interests: The author(s) declared that no potential competing interests exist.

\section{Abstract}

Background: Electronic Nicotine Delivery Systems (ENDS) have seen increased use in the past several years with no clear guide for healthcare providers to help motivated users to quit.

Case Study: 25-year-old African American male who had been vaping since 2015 started using nicotine replacement therapy and received behavioral support by a pharmacist to help quit.

Conclusion: Nicotine replacement therapy paired with behavioral counseling could be beneficial to ENDS users by reducing withdrawal symptoms and helping them quit.

\section{Background}

Electronic Nicotine Delivery Systems (ENDS) have seen increased use in the past several years. ${ }^{1-3}$ Despite the influx of users, there is still very little data to support how to aid patients with cessation attempts. ENDS have also been used in several studies as cessation aids for cigarettes, but these studies have not been conclusive. ${ }^{4-6}$ Furthermore, studies indicate that many people who switch to ENDS continue to use their ENDS device long-term and never completely cease 
using nicotine products. ${ }^{4}$ With recent increases in vaping related illness across the country, this proves concerning. ${ }^{7}$ Additionally, while cessation aides are well studied in traditional cigarette cessation, there are currently only a few case reports exploring the use of nicotine replacement therapy (NRT) and varenicline for ENDS cessation., 8 This can leave clinicians wondering what sort of cessation aid is best for their patients, along with how they should apply cessation aid directions based on a number of cigarettes smoked per day or time to first cigarette to a device with a much less standard pattern of use.

\section{Case Description}

The case described is one participant of a larger study which is a prospective, 3-arm, parallel group randomized study designed to develop and evaluate the success of ENDS cessation protocols. The study was not blinded. Individuals in the nicotine replacement therapy (NRT) arm were assessed using a version of the Fagerstrom Test for Nicotine Dependence modified to relate to vaping practices (Figure 1). They were then assigned NRT based on the results of their Fagerstrom Test (Figure 2).

The individual described in this case study was a 25-year-old African American male who had been vaping since 2015. He noted no other tobacco use. When he began the study, he was vaping with $35 \mathrm{mg} / \mathrm{mL}$ e-juice and using approximately $1 \mathrm{~mL}$ of e-juice per day. He had attempted to quit ENDS two times in the past, quitting for 1 month the first time and 10 days the second. Using the modified Fagerstrom test, the individual received a score of 6, indicating moderate nicotine dependence. Based on the modified Fagerstrom test score, he was started on combination NRT with a $21 \mathrm{mg} /$ day patch and $2 \mathrm{mg}$ lozenges. Together, the pharmacist and participant decided on a quit date and finalized a quit plan. He was instructed to use 1 patch per day and always use a lozenge in the morning, as he frequently had morning cravings. He was to use additional lozenges as needed for cravings throughout the day. The pharmacist counseled the participant on proper use of NRT lozenges and patches, advised of possible side effects, and discussed possible nicotine withdrawal symptoms. The patient was encouraged to get rid of all vaping supplies to help with the temptation to keep vaping. A follow-up phone call was scheduled 6 days after his first appointment, since nicotine withdrawal symptoms are most intense within 3-7 days of quitting. At this call, he was experiencing mild withdrawal symptoms leading to cravings to vape that he was combating with the lozenge. He was using about 12 lozenges per day. He noticed that he was breathing a little better and discussed wanting to work out more. The pharmacist discussed the importance of behavior change to replace the habit of vaping and agreed that getting into a normal routine of exercise would be a great option for this. At his 2-week phone check-in, the individual said that he had not been experiencing as many cravings and that working on a consistent daily routine and exercising was helping him to be successful in quitting. He was using a $21 \mathrm{mg}$ patch along with seven $2 \mathrm{mg}$ lozenges per day while in class and five $2 \mathrm{mg}$ pieces of gum per day when out of class.

At his 4-week in-person appointment, the individual had 10 nicotine patches left, indicating that he was not compliant with the patches due to some itching where the patch was applied, but he was still consistently using lozenges and gum. $\mathrm{He}$ was using about 9 pieces per day and noted that this was helping him to be successful on the quit attempt. He had not 
noticed any changes in mood after getting past the first week, and felt that his appetite and concentration were better, and he was coughing less. He asked about using only nicotine gum since he thought this would help more with the "habit" that was formed while vaping. At the end of the appointment, he was instructed to step down to $14 \mathrm{mg}$ nicotine patches and up to $4 \mathrm{mg}$ gum. Together, the pharmacist and participant decided he would only use patches on long days with work and could use up to 2 pieces of gum on those days. On other days, he was going to use gum more frequently, up to 6 pieces per day. At his 6-week check-in call, he noted that he had used 11 patches and followed his regimen for the gum. He also noted that he felt he had more energy throughout the day and continued to cough less.

At his 8-week in-person appointment, he noted similar improvements to his health, and that he had used 26 (out of 28) 14 mg nicotine patches. He was using an average off 4-5 pieces of gum throughout the day. He felt that the study had given him the push he needed to quit and that getting rid of his vaping supplies when the study began was extremely helpful. After discussion with the pharmacist, he decided to use only gum for the remainder of his quit attempt. He was going to begin tapering off the gum by using 5 pieces a day for week 9,4 pieces a day for week 10, 3 pieces a day for week 11 , and 1 piece a day for week 12, after which he would quit completely. At his 10-week follow-up call, he was successfully using 1-2 pieces of gum in the day, and was going to drop to only 1 piece per day the following week.

At his final appointment, the individual realized that getting good sleep and keeping a routine were very beneficial in keeping him from vaping during the study. He planned to continue to do both of these in order to continue to be successful. He noted that his biggest challenge in quitting was the withdrawal symptoms he experienced the first week of the study, but felt there were few barriers in his way now to remain vape- and nicotine-free. He also noted that the NRT was very beneficial to help him get past his cravings.

\section{Conclusion}

This case study clearly shows that individuals who are looking to quit vaping are plagued by withdrawal symptoms that can prevent them from being successful. The unique accessibility of ENDS makes feeding these cravings and relapsing very easy. In this specific case, NRT and behavioral counseling helped the individual participating in the study to get past his withdrawal symptoms and successfully quit. Nicotine replacement therapy paired with behavioral counseling could be beneficial to ENDS users and needs to be studied more in order to establish an effective protocol to assist healthcare providers guiding users in quitting.

\section{Acknowledgements}

This study was funded by a generous grant from Michigan Pharmacists Foundation and Ferris State University Faculty Research Grant.

\section{References}

1. Cullen KA, Gentzke AS, Sawdey MD, et al. e-Cigarette Use Among Youth in the United States, 2019. JAMA. 2019;322(21):2095-2103. doi:10.1001/jama.2019.18387 
2. Centers for Disease Control and Prevention / National Center for Chronic Disease Prevention and Health Promotion. National Youth Tobacco Survey (NYTS). Smoking and Tobacco Use.

http://www.cdc.gov/tobacco/data_statistics/surveys/nyts/. Published May 22, 2018. Accessed November 16, 2018.

3. Hammond D, Reid JL, Rynard VL, et al. Prevalence of vaping and smoking among adolescents in Canada, England, and the United States: repeat national cross sectional surveys. BMJ. 2019;365:I2219. doi:10.1136/bmj.I2219

4. Hajek P, Phillips-Waller A, Przulj D, et al. A Randomized Trial of E-Cigarettes versus Nicotine-Replacement Therapy. New England Journal of Medicine. 2019;0(0):null. doi:cdc

5. National Academies report on e-cigarettes highlights need for more research, guidance. Pharmacy Today. 2018;24(6):28. doi:10.1016/j.ptdy.2018.05.020

6. El Dib R, Suzumura EA, Akl EA, et al. Electronic nicotine delivery systems and/or electronic non-nicotine delivery systems for tobacco smoking cessation or reduction: a systematic review and meta-analysis. BMJ Open.

2017;7(2):e012680. doi:10.1136/bmjopen-2016-012680

7. CDC updates EVALI guidance for health care providers as flu activity increases nationally | CDC Online Newsroom | CDC. https://www.cdc.gov/media/releases/2019/p1119-evali-guidance.html. Published November 19, 2019. Accessed November 20, 2019.

8. Silver B, Ripley-Moffitt C, Greyber J, Goldstein AO. Successful use of nicotine replacement therapy to quit e-cigarettes: Lack of treatment protocol highlights need for guidelines. Clin Case Rep. 2016;4(4):409-411. doi:10.1002/ccr3.477 9. Barkat SS, Tellier SM, Eloma AS. Varenicline for cessation from nicotine-containing electronic cigarettes. American Journal of Health-System Pharmacy. 2019;76(23):1894-1895. doi:10.1093/ajhp/zxz218 